\title{
Endorectal balloon (ERB) in helical tomotherapy (HT) for localized prostate cancer: a case report of dosimetric analysis
}

\author{
Seung-Gu Yeo^, Kwang Hwan Cho \\ Department of Radiation Oncology, Soonchunhyang University Bucheon Hospital, Soonchunhyang University College of Medicine, Bucheon, \\ Republic of Korea \\ Correspondence to: Seung-Gu Yeo. Department of Radiation Oncology, Soonchunhyang University Bucheon Hospital, Soonchunhyang University \\ College of Medicine, 170 Jomaru-ro, 14584 Bucheon, Republic of Korea. Email: md6630@schmc.ac.kr.
}

\begin{abstract}
Radiation therapy (RT) for localized prostate cancer yields oncological outcomes similar to those following radical prostatectomy, but is associated with more anorectal toxicity. An endorectal balloon (ERB) has been utilized to decrease the incidental dose to the rectal wall. However, few studies analyzed whether the ERB can further spare the rectum in helical tomotherapy (HT), which by itself can be used to treat prostate cancer while minimizing irradiation of surrounding critical tissues. Here, we report a 64-year-old man with pathologically proven prostate adenocarcinoma (stage T2cN0M0). He underwent definitive RT using HT with a hypofractionated scheme of 70 Gy in 28 fractions. Simulation CT was performed twice: with and without ERB application. The ERB was filled with $70 \mathrm{~mL}$ of air. Two intensity-modulated RT (IMRT) plans were generated for each CT image set (with and without ERB) and compared about the dose to the anorectum. The rectal volume receiving $\geq 40$ Gy (V40Gy) was reduced from $43.4 \%$ to $34.6 \%$ with ERB use (20.3\% reduction). This reduction rate increased continuously up to V70Gy (48.2\% reduction). The anal volume reduction was approximately 50\% from V5Gy to V15Gy. The patient tolerated all ERB insertions well and there were no severe acute toxicities. ERB had a further anorectal-sparing effect in this case of prostate cancer treated by highly conformal HT, beyond the generally recommended dose-volume constraints of hypofractionated IMRT.
\end{abstract}

Keywords: Prostatic neoplasms; radiotherapy; intensity-modulated (IM); case report

Submitted May 06, 2021. Accepted for publication Jul 14, 2021.

doi: $10.21037 /$ tcr-21-777

View this article at: https://dx.doi.org/10.21037/tcr-21-777

\section{Introduction}

Radiotherapeutic and surgical treatments provide similar oncological outcomes for localized prostate cancer. Therefore, their different side effect profiles that impact the quality of life often become important factors in treatment selection (1). A recent review article stated that radical prostatectomy is associated more with urinary incontinence and erectile dysfunction, while radiation therapy (RT) is associated more with worsening bowel function (2). Even with modern RT techniques, such as intensity-modulated RT (IMRT) and imaging guidance, late anorectal toxicity rates varying from $5 \%$ to $65 \%$ have been reported (3).

Endorectal balloon (ERB) insertion is a minimally invasive method for reducing unnecessary anorectal irradiation in prostate RT. The ERB pushes the posterior and lateral rectal walls out of the high-dose region and thereby lower anorectal toxicity (4). It also restricts prostate motion by minimizing variations in rectal position and pushing the prostate toward the pubic bone (5). However, with the advent of modulated-intensity techniques with imaging guidance, ERB may provide little additional benefit. The dosimetric advantages of ERB clearly shown by three-dimensional conformal RT (3DCRT) were not always

\footnotetext{
$\wedge$ ORCID: 0000-0003-1371-1381.
} 
reproduced in IMRT studies (6). Furthermore, helical tomotherapy (HT) has a higher spatial resolution than stepand-shoot IMRT, due to the use of a single leaf bank with a backup collimator to allow continuous adjustment of the radiation field (7). To date, few studies of HT for prostate cancer patients have included dosimetric analysis with ERB application.

Here, we present a dosimetric analysis of the anorectalsparing effect of ERB in a patient with localized prostate cancer managed with HT. We present the following case in accordance with the CARE reporting checklist (available at https://dx.doi.org/10.21037/tcr-21-777).

\section{Case presentation}

A 64-year-old man presented with an elevated prostatespecific antigen (PSA) level detected during a medical checkup. He had no specific symptoms or comorbidities. The serum PSA level was $8.16 \mathrm{ng} / \mathrm{mL}$. Transrectal ultrasound-guided prostate biopsy demonstrated adenocarcinoma involving the left lobe. The Gleason score was $3+4=7$. Magnetic resonance imaging showed a $2 \mathrm{~cm}$ mass involving the apical anterior right transitional zone and several focal diffusion-restricted spots in the bilateral peripheral zone. Seminal vesicle invasion and abnormal enlargement of pelvic lymph nodes were not observed. No specific findings were seen on whole-body bone scan or chest X-ray. The tumor was diagnosed clinically as T2cN0M0 according to the American Joint Committee on Cancer staging system, 8th ed. The institutional review board of Soonchunhyang University Bucheon Hospital waived the requirement for approval as this is a retrospective case report. All procedures performed in studies involving human participants were in accordance with the ethical standards of the institutional and/or national research committee(s) and with the Helsinki Declaration (as revised in 2013). Written informed consent was obtained from the patient.

The patient underwent definitive RT with HT. For simulation, pelvic CT was performed with a $2 \mathrm{~mm}$ slice thickness in the supine position with the arms above the head (SOMATOM Confidence; Siemens Healthcare, Erlangen, Germany). He was instructed to drink $300 \mathrm{~mL}$ of water 1 hour prior to the simulation and each treatment. An anti-flatulence diet was also recommended. The patient straightened his legs with ankle immobilization. After CT without the balloon, the rectal balloon was applied. A deflated rectal catheter capped with a latex condom and lubricating jelly was gently inserted into the anus in the decubitus position. The balloon was inflated slowly with $70 \mathrm{~mL}$ of air and pulled back toward the anal canal. The scale on the catheter was recorded so that each insertion would be made at the same depth. Then, contrastenhanced CT was performed. Both CT image sets were transferred to a Precision planning system (Accuray Inc., Sunnyvale, CA, USA).

Target volumes and adjacent normal structures, including the bladder, rectum, anal canal, and femoral heads, were outlined on each axial image in the two CT sets. The clinical target volume (CTV) was defined as the prostate gland and proximal $1 \mathrm{~cm}$ of the seminal vesicles. The planning target volume (PTV) was defined as the CTV plus an additional $5 \mathrm{~mm}$ area in all directions, except $3 \mathrm{~mm}$ posteriorly. The entire bladder and rectal volume, including the wall and lumen, was regarded as the organ at risk. The anal canal was contoured from the anal verge to the slice below the balloon. On CT without ERB, it was contoured to include the distal $3 \mathrm{~cm}$ of the anorectum (8). The rectum was contoured from the anal canal to the rectosigmoid flexure. A moderate hypofractionation scheme of $70 \mathrm{~Gy}$ in 28 fractions (2.5 Gy/fraction) was prescribed. The criterion for planning the PTV was that at least $95 \%$ should be covered by $95 \%$ of the prescribed dose. The dose-volume constraints for organs at risk followed the Radiation Therapy Oncology Group 0415 protocol and were the same for both plans (9). The patient was treated using a Radixact treatment system (Accuray) with ERB application. Megavoltage CT was performed daily before treatment to verify proper patient setup and ERB localization. A daily disposable ERB was used for hygiene reasons. Two plans, generated on each CT set with or without ERB, were compared about the dose to the anorectum.

Figures 1,2 show RT plans and dose-volume histograms, respectively, according to ERB use. The rectal volume was $35.9 \mathrm{~mL}$ without the ERB and $115.1 \mathrm{~mL}$ with the ERB. Tables 1,2 list the intermediate to high dose parameters of the rectum and anal canal, respectively, according to ERB use. The rectal volume receiving $\geq 40$ Gy (V40Gy) was reduced from $43.4 \%$ to $34.6 \%$ with ERB use (20.3\% reduction). This reduction increased continuously up to V70Gy (reduction of $48.2 \%)$. The anal volume reduction was approximately $50 \%$ from V5Gy to V15Gy. All dose-volume parameters were decreased after ERB application, and the reduction rate was higher on the anal canal, although the absolute dose was relatively low. Absolute rectal volume receiving $\geq 70$ Gy was $4.08 \mathrm{~mL}$ without the ERB, and $6.75 \mathrm{~mL}$ 

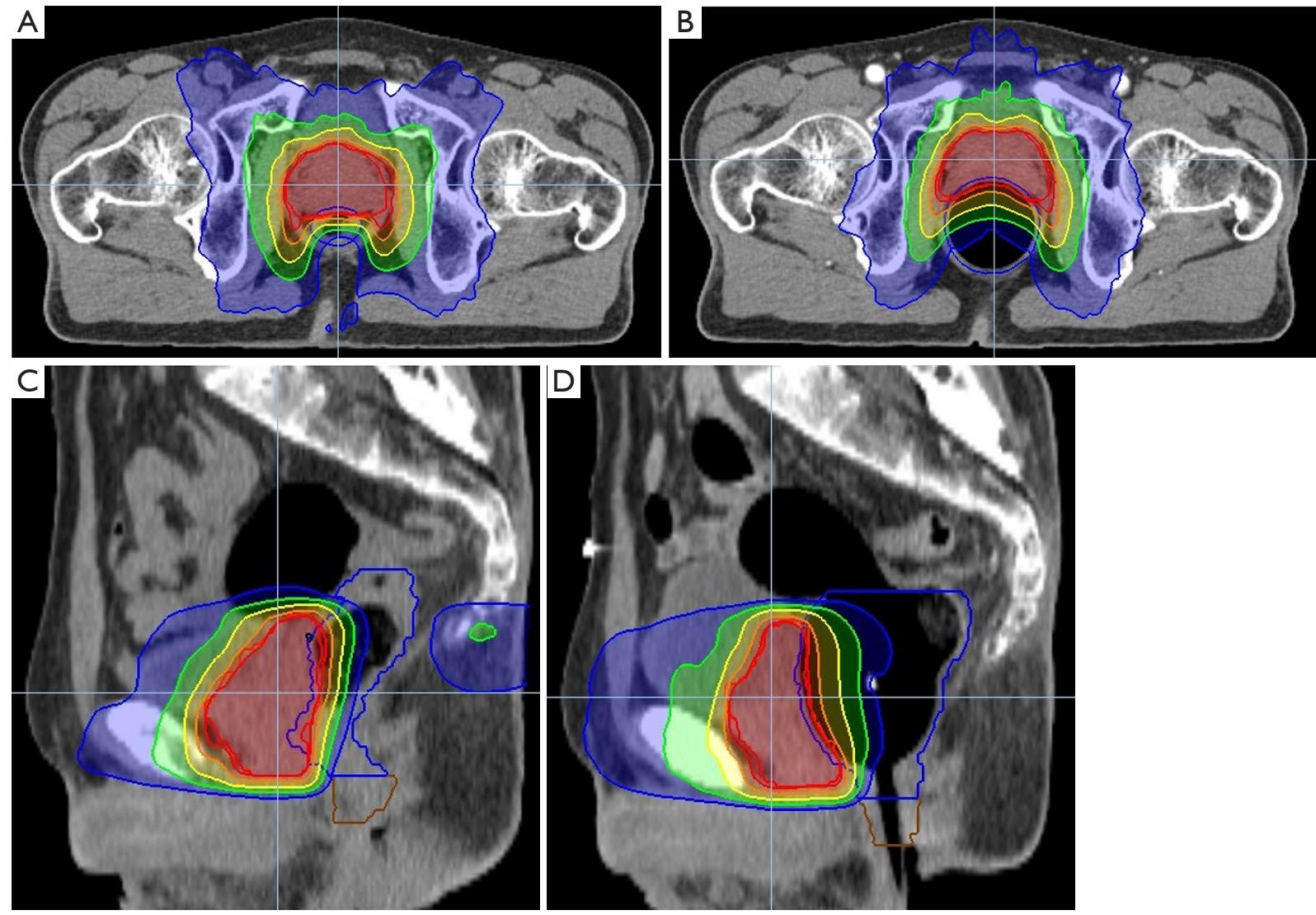

Figure 1 Comparison of treatment plans according to ERB use. (A) Axial view without ERB. (B) Axial view with ERB. (C) Sagittal view without ERB. (D) Sagittal view with ERB (red, planning target volume; blue, rectum; brown, anal canal; others are radiotherapy plan isodose lines). ERB, endorectal balloon.

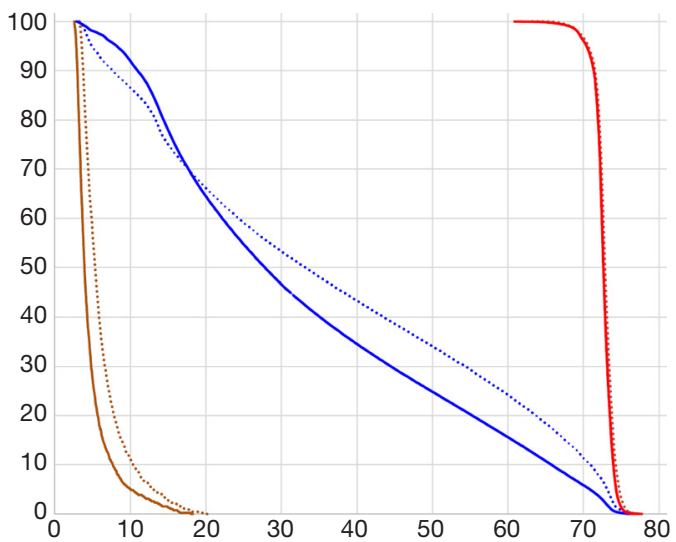

Figure 2 Dose-volume histograms according to ERB use. Bin doses (Gy) are indicated along the horizontal axis, and structure volumes (\%) are on the vertical. Solid and dotted lines represent ERB application and non-application, respectively (red, planning target volume; blue, rectum; brown, anal canal). ERB, endorectal balloon.
Table 1 Rectal dose-volume parameters according to ERB use

\begin{tabular}{|c|c|c|c|}
\hline \multirow{2}{*}{ Rectum } & \multicolumn{2}{|c|}{ ERB } & \multirow{2}{*}{ Reduction (\%) } \\
\hline & No & Yes & \\
\hline V40Gy $(\%)^{*}$ & 43.4 & 34.6 & 20.3 \\
\hline V50Gy (\%) & 34.1 & 24.8 & 27.3 \\
\hline V60Gy (\%) & 24.2 & 15.6 & 35.5 \\
\hline V70Gy (\%) & 11.4 & 5.9 & 48.2 \\
\hline Mean (Gy) & 36.77 & 33.32 & 9.4 \\
\hline
\end{tabular}

*, rectal volume receiving $\geq 40$ Gy. ERB, endorectal balloon. 
Table 2 Anal dose-volume parameters according to ERB use

\begin{tabular}{|c|c|c|c|}
\hline \multirow{2}{*}{ Anal canal } & \multicolumn{2}{|c|}{ ERB } & \multirow{2}{*}{$\begin{array}{c}\text { Reduction } \\
(\%)\end{array}$} \\
\hline & No & Yes & \\
\hline V5Gy $(\%)^{\star}$ & 56.7 & 27.4 & 51.7 \\
\hline V10Gy (\%) & 11.2 & 5.1 & 54.5 \\
\hline V15Gy (\%) & 2.8 & 1.4 & 50.0 \\
\hline Mean (Gy) & 6.30 & 4.78 & 24.1 \\
\hline
\end{tabular}

*, anal volume receiving $\geq 5$ Gy. ERB, endorectal balloon.

with the ERB. The patient completed 28 fractionated treatments with no rest and tolerated ERB insertions well. There were no severe acute toxicities.

\section{Discussion}

IMRT has become standard practice for prostate cancer, replacing 3DCRT. As irradiation to surrounding critical structures is decreased with IMRT, the ERB-induced dosimetric advantage shown by 3 DCRT needed to be evaluated for IMRT. Reported outcomes are somewhat mixed. van Lin et al. (10) compared the use of 40, 80, and $100 \mathrm{~mL}$ ERB with no ERB in patients undergoing fourfield 3DCRT and step-and-shoot IMRT. In 3DCRT, ERB significantly reduced the measured rectal wall mean dose, V50Gy, and V70Gy, and 80 and $100 \mathrm{~mL}$ ERB showed better performance than $40 \mathrm{~mL}$ ERB. However, IMRT did not significantly reduce any rectal wall dose parameters for all three ERBs. In contrast to this study, Patel et al. (11) used a $60 \mathrm{~mL}$ ERB only in IMRT and found that ERB reduced the rectal wall V60Gy, V65Gy, and V70Gy. They showed that the rectal dose-sparing effect in 3DCRT with a balloon was comparable to IMRT without a balloon, and inclusion of a balloon had a further rectal-sparing effect in IMRT. Both of the above studies used a step-andshoot IMRT technique with five or seven coplanar photon beams.

In addition to step-and-shoot IMRT, a variety of novel IMRT delivery methods have been introduced, including volumetric modulated arc therapy and HT. A dosimetric comparison (without ERB) of 3DCRT, step-and-shoot IMRT, volumetric modulated arc therapy, and HT in prostate cancer indicated that HT showed the best rectal sparing ability (7). Questions arose whether further dosimetric benefits can be achieved using ERB in HT because HT itself has a better rectal sparing effect than other IM techniques.
To our knowledge, this is the first study to show the dosimetric advantage of ERB even in HT for prostate cancer, although our findings were confined to a single case and no statistical analyses were possible. A limitation that the air cavity created by ERB can perturb accurate dose calculation also necessitates more patient data (12).

We also separately analyzed the dose to the anal canal and observed a greater sparing effect of ERB in the anal canal than the rectum, although the absolute anal dose was relatively small. Smeenk et al. (8) demonstrated an anal wallsparing effect of ERB in both 3DCRT and IMRT. They considered this effect to be due to the increase in distance between the PTV and anal wall caused by the anterior shift of the prostate by the balloon and to the shaft of the ERB pushing the posterolateral part of the anal wall away from the irradiated volume.

Prostate RT is associated with a higher rate of anorectal toxicity compared to surgical resection (2). Irradiation of the whole circumference of the viscus, which has radial distensibility, would lead to stricture and an increased likelihood of bleeding when substances pass through. However, when only a small part of the hollow viscus receives high-dose irradiation, the remainder maintains radial distensibility. In this case, pressure from passing substances will be low, and the risks of obstruction and bleeding are lower (13). Meanwhile, a patient selfassessment indicated that patients appeared to be most bothered by soiling, fecal loss, and urgency than by rectal bleeding or mucus discharge (14). Anorectal toxicity has a great impact on patients' quality of life. Complaints of fecal urgency were associated with decreased external anal sphincter function (15). Dose-volume constraints in the guidelines were not derived using bowel quality of life assessments, and there is no precise dose threshold above which rectal toxicity will occur $(16,17)$. Thus, the dosevolume reduction shown in this case, including a greater reduction in the anal canal, suggests the clinical benefit of ERB application in HT for prostate cancer.

As a higher dose per fraction is prescribed for prostate cancer, including hypofractionated IMRT and stereotactic ablative RT, ERB may be more beneficial because a high dose per fraction can expose the rectum to doses beyond its repair capability (17). The incidental dose to the rectal wall can be reduced by using a software-based technique, i.e., IM plan optimization, but this has limitations due to the proximity of the rectum to the target. The use of supplementary devices, such as ERB and injectable spacer gels between the prostate and rectum, can reduce rectal 
dose and potentially also rectal toxicity (18). The relatively novel technique of using spacers to separate the anterior rectal wall from the prostate was reported to outperform the ERB about rectal sparing parameters in stereotactic ablative RT (17). However, the anal wall-sparing effect of spacers has not yet been specifically addressed (3).

In conclusion, although this report is limited to a single case such that more data and statistical analyses are required, in highly conformal HT for prostate cancer, ERB appears to provide an extra anorectal-sparing effect beyond the generally recommended dose-volume constraints of hypofractionated IMRT.

\section{Acknowledgments}

Funding: This work was supported by the Soonchunhyang University Research Fund.

\section{Footnote}

Reporting Checklist: The authors have completed the CARE reporting checklist. Available at https://dx.doi. org/10.21037/tcr-21-777

Conflicts of Interest: Both authors have completed the ICMJE uniform disclosure form (available at https://dx.doi. org/10.21037/tcr-21-777). The authors have no conflicts of interest to declare.

Ethical Statement: The authors are accountable for all aspects of the work in ensuring that questions related to the accuracy or integrity of any part of the work are appropriately investigated and resolved. All procedures performed in studies involving human participants were in accordance with the ethical standards of the institutional and/or national research committee(s) and with the Helsinki Declaration (as revised in 2013). Written informed consent was obtained from the patient for publication of this case report and accompanying images. A copy of the written consent is available for review by the editorial office of this journal.

Open Access Statement: This is an Open Access article distributed in accordance with the Creative Commons Attribution-NonCommercial-NoDerivs 4.0 International License (CC BY-NC-ND 4.0), which permits the noncommercial replication and distribution of the article with the strict proviso that no changes or edits are made and the original work is properly cited (including links to both the formal publication through the relevant DOI and the license). See: https://creativecommons.org/ licenses/by-nc-nd/4.0/.

\section{References}

1. Martin NE, D'Amico AV. Progress and controversies: Radiation therapy for prostate cancer. CA Cancer J Clin 2014;64:389-407.

2. Taylor JM, Chen VE, Miller RC, et al. The Impact of Prostate Cancer Treatment on Quality of Life: A Narrative Review with a Focus on Randomized Data. Res Rep Urol 2020;12:533-46.

3. Smeenk RJ, van Lin EN. Application of anorectal sparing devices in prostate radiotherapy. Radiother Oncol 2013;106:155-6.

4. Smeenk RJ, Teh BS, Butler EB, et al. Is there a role for endorectal balloons in prostate radiotherapy? A systematic review. Radiother Oncol 2010;95:277-82.

5. Afkhami Ardekani M, Ghaffari H, Navaser M, et al. Effectiveness of rectal displacement devices in managing prostate motion: a systematic review. Strahlenther Onkol 2021;197:97-115.

6. Serrano NA, Kalman NS, Anscher MS. Reducing rectal injury in men receiving prostate cancer radiation therapy: current perspectives. Cancer Manag Res 2017;9:339-50.

7. Tsai CL, Wu JK, Chao HL, et al. Treatment and dosimetric advantages between VMAT, IMRT, and helical tomotherapy in prostate cancer. Med Dosim 2011;36:264-71.

8. Smeenk RJ, van Lin EN, van Kollenburg P, et al. Anal wall sparing effect of an endorectal balloon in $3 \mathrm{D}$ conformal and intensity-modulated prostate radiotherapy. Radiother Oncol 2009;93:131-6.

9. Morgan SC, Hoffman K, Loblaw DA, et al. Hypofractionated Radiation Therapy for Localized Prostate Cancer: Executive Summary of an ASTRO, ASCO, and AUA Evidence-Based Guideline. Pract Radiat Oncol 2018;8:354-60.

10. van Lin EN, Hoffmann AL, van Kollenburg P, et al. Rectal wall sparing effect of three different endorectal balloons in 3D conformal and IMRT prostate radiotherapy. Int J Radiat Oncol Biol Phys 2005;63:565-76.

11. Patel RR, Orton N, Tomé WA, et al. Rectal dose sparing with a balloon catheter and ultrasound localization in conformal radiation therapy for prostate cancer. Radiother Oncol 2003;67:285-94. 
12. Hardcastle N, Metcalfe PE, Rosenfeld AB, et al. Endorectal balloon cavity dosimetry in a phantom: performance under IMRT and helical tomotherapy beams. Radiother Oncol 2009;92:48-56.

13. Cho JH, Lee CG, Kang DR, et al. Positional reproducibility and effects of a rectal balloon in prostate cancer radiotherapy. J Korean Med Sci 2009;24:894-903.

14. Koper PC, Jansen P, van Putten W, et al. Gastro-intestinal and genito-urinary morbidity after $3 \mathrm{D}$ conformal radiotherapy of prostate cancer: observations of a randomized trial. Radiother Oncol 2004;73:1-9.

15. Krol R, Hopman WP, Smeenk RJ, et al. Increased rectal wall stiffness after prostate radiotherapy: relation with fecal urgency. Neurogastroenterol Motil 2012;24:339-e166.

Cite this article as: Yeo SG, Cho KH. Endorectal balloon (ERB) in helical tomotherapy (HT) for localized prostate cancer: a case report of dosimetric analysis. Transl Cancer Res 2021;10(9):4250-4255. doi: 10.21037/tcr-21-777
16. Quinn TJ, Daignault-Newton S, Bosch W, et al. Who Benefits From a Prostate Rectal Spacer? Secondary Analysis of a Phase III Trial. Pract Radiat Oncol 2020;10:186-94.

17. Jones RT, Hassan Rezaeian N, Desai NB, et al. Dosimetric comparison of rectal-sparing capabilities of rectal balloon vs injectable spacer gel in stereotactic body radiation therapy for prostate cancer: lessons learned from prospective trials. Med Dosim 2017;42:341-7.

18. Miller LE, Efstathiou JA, Bhattacharyya SK, et al. Association of the Placement of a Perirectal Hydrogel Spacer With the Clinical Outcomes of Men Receiving Radiotherapy for Prostate Cancer: A Systematic Review and Meta-analysis. JAMA Netw Open 2020;3:e208221. 